\title{
Learning to 'think systems'
}

\author{
Nanoscience can play an important role in addressing a number of societal challenges, but, as \\ Rein V. Ulijn and Elisa Riedo explain, research training needs to evolve.
}

$\mathrm{O}$ ver the last decade, nanoscience has increasingly moved from concepts to applications, and the field is poised to have a significant economic and societal impact through areas such as urbanization, energy generation, climate change and healthcare. There is, therefore, an urgent need to train a workforce that is both well versed in fundamental nanoscience and adequately equipped to deal with its applications. This means that the next generation of nanoscience students and researchers will need to learn to navigate the ways in which nanoscience can interact with, and impact on, other disciplines and society, as well as learning about their own (already diverse) research field. To address these challenges, 'systems thinking' will be required.

Systems thinking involves the translation of concepts and ideas across disciplinary barriers, and also across length scales, from nano to macro to global; it involves a consideration of how fundamental insights connect to applications, and how various theoretical and experimental parts connect and can be integrated into a functional whole. Following biologists and chemists, nanoscientists have recently started to appreciate the value of a systems approach ${ }^{1-3}$, but such ideas have received only limited attention in nanoscience curricula so far.

At the City University of New York (CUNY) Advanced Science Research Center (ASRC) in Manhattan, New York, we are developing ways in which to train nanoscientists to become comfortable with connecting their discipline to societal challenges. The ASRC is housed in a purpose-built building with space designed to address research challenges with approaches that transcend disciplines and scales. The centre is organized around five thematic areas - nanoscience, photonics, structural biology, neuroscience and environmental science - distributed across the floors of the building (Fig. 1). The ASRC provides an environment in which to develop ways of training students and scientists that incorporate a better appreciation of scale and connectivity between disciplines, and is, therefore, a unique test bed for new didactic and research approaches to applied nanoscience.

For example, while nanoscience graduate students on the first floor of the ASRC study the properties of water in nanoscale confinement ${ }^{4}$ and aqueous phase molecular assemblies ${ }^{5}$, environmental science colleagues on the fifth floor study global water management challenges ${ }^{6}$. Students from across these floors are encouraged to interact in order to develop new ways to tackle global water issues; our aim, in other words, is to integrate researchers who view a single topic (in this case, aqueous systems) through disciplinary lenses that can be up to 15 orders of magnitude apart in length scales. Together, these students are developing new nanomaterials for water purification, as well as nanotech-based sensing systems that enable data collection in large-scale field applications.

Our objective is to incorporate elements of systems thinking into all areas of the ASRC to create a truly integrated research culture. It starts with informal weekly coffee meetings,

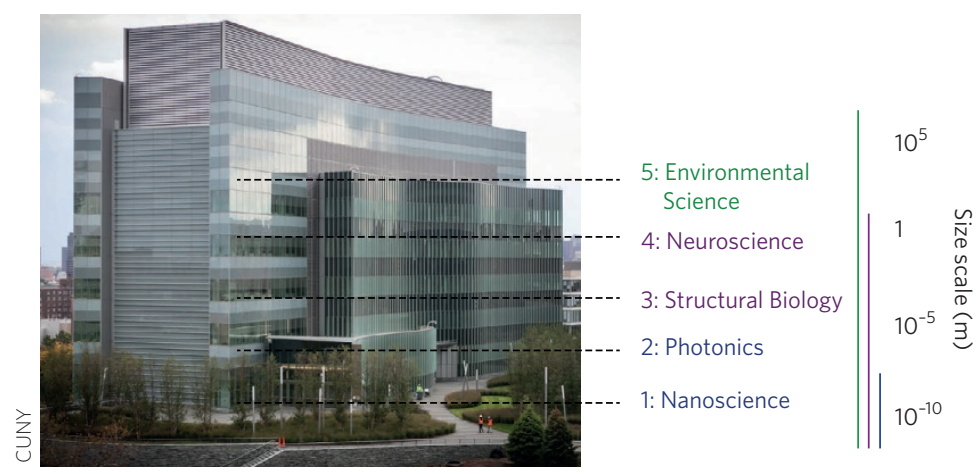

Figure 1 | The ASRC in New York, which is organized around five thematic areas that cover 15 orders of magnitude in length scale.

in which one scientist addresses staff and students from all across the building.

Of course, constructive thinking about the wider context of a scientific problem first requires a firm understanding of your own discipline, and it is critical not to dilute this aspect in any training programme. Therefore, degrees of engagement vary depending on training level and experience. For example, undergraduate students from different subjects are exposed to the role that nanoscience can play across disciplines through tours of the laboratories and site visits, which include demonstrations and short talks by graduate students. However, graduate students from the physics, engineering, biology and chemistry programmes gain hands-on experience in nanoscience characterization and fabrication by using high-end electron microscopes and nanofabrication facilities.

The aims of the ASRC are also encased in the mission of CUNY: to provide high-quality, affordable education for students, and to contribute significantly to the future workforce of New York City. The ASRC only opened its doors two years ago, but CUNY has a tremendous history, with alumni that include thirteen Nobel laureates, many of whom are children of immigrants to the country. Building on this, we hope to take advantage of the unique cross-disciplinary setting offered by the ASRC, and provide students and young researchers with the joined-up training that the future of nanoscience demands.

REIN V. ULIJN is the Director of the Nanoscience Initiative at CUNY ASRC and Professor of Chemistry at Hunter College. ELISA RIEDO is Professor of Physics in the Nanoscience Initiative at CUNY ASRC and Professor of Physics at the City College of New York.

e-mail: rein.ulijn@asrc.cuny.edu; elisa.riedo@asrc.cuny.edu

References

1. Grzybowski, B. A. \& Huck, W. T. S. Nat. Nanotech 11, 585-592 (2016).

2. Mattia, E. \& Otto, S. Nat. Nanotech. 10, 111-119 (2015).

3. Matlin, S. A., Mehta, G., Hopf, H. \& Krief, A. Nat. Chem. 8, 393-398 (2016).

4. Ortiz-Young, D., Chiu, H.-C., Kim, S., Voitchovsky, K. \& Riedo, E. Nat. Commun. 4, 2482 (2013).

5. Frederix, P. W. J. M. et al. Nat. Chem. 7, 30-37 (2015).

6. Vörösmarty, C. J., Hoekstra, A. Y., Bunn, S. E., Conway, D. \& Gupta, J. Science 349, 478-479 (2015). 